\title{
Is there a Role for Long Chain Omega-3 (N-3) Fatty Acids in Reversal of Malnutrition Inflammation Syndrome among Hemodialysis Patients?
}

\section{Mary Harris*}

Department of Food Science and Human Nutrition, Colorado State University, USA

Mortality and cardiovascular morbidity among hemodialysis patient's remains unacceptably high in the US. Age related mortality is two to three times that of the general population. Although inflammation is a common feature of cardiovascular disease in both end-stage chronic kidney disease and non-dialysis populations, significant differences exist. In chronic kidney disease (CKD), inflammation is a hallmark of malnutrition-inflammation syndrome (MIS), characterized by failure to thrive, erythropoietin (EPO) resistant anemia and hypoalbuminemia. Numerous factors contribute to inflammation; among these are interactions of blood components with the AV fistula or dialysis tubing and the presence of failed allografts. Inflammation is the prime target for intervention in patients with MIS but currently no medical intervention has been effective for prevention and management of the condition. Given the increasing prevalence of chronic kidney disease and the association of MIS with CVD mortality it is clear that a treatment is urgently needed. Whereas conventional dietary treatments have failed to manage MIS, increasing long chain n-3 fatty acid intake from fish oil supplements or the consumption of fatty fish may prove to be of benefit. Long chain n-3 fatty acids, eicosapentaenoic acid (EPA) and docosahexaenoic acid (DHA) modulate inflammation as precursors to resolvins and protectins, by down regulating the overall synthesis of eicosanoids and by shifting synthesis from the pro-inflammatory 2-series prostaglandins (PGs) and 4-series leukotrienes (LTs) toward the less potent 3-series PGs and 5-series LTs. Several studies have shown that high levels of inflammatory biomarkers, particularly those with the highest predictive value for CVD in dialysis patients, C-reactive protein and IL-6, may be reduced by supplementation with fish oil fatty acids. Randomized control trials [1-7] with fish oil supplements providing 1.8 to $2.9 \mathrm{~g}$ of EPA and DHA/day have demonstrated beneficial effects on inflammatory biomarkers but generally failed to produce consistent improvements in clinical outcomes including BMI, body weight, malnutrition-inflammation scores, serum albumin, transferrin and hematocrit levels. A decrease in pro-inflammatory IL-6, tumor necrosis factor and CRP corresponding to increased IL-10 and a shift in anti-inflammatory: pro-inflammatory cytokine ratio has been demonstrated. While this is encouraging, treatment resulted in little improvement in serum triglycerides as a CVD risk indicator [8]. To date, clinical intervention trials been small and treatment times were short. In most studies, supplementation periods averaged 4 months and this may not have been an adequate duration to recognize clinical benefits. Additionally, intervention for MIS may be more effective if initiated at earlier stages of CKD. Our laboratory conducted an longer term intake study in felines with stage 2 and 3 CKD and demonstrated a positive association between the long chain $n-3$ fatty acids (EPA and DHA) and serum albumin and body condition score [9]. Long chain n-3 fatty acids were negatively correlated with serum alpha amyloid (SAA), a more sensitive biomarker of inflammation in felines than CRP. In this model, both SAA and the acute phase protein, acid 1 alpha glycoprotein, were inversely correlated with hemoglobin, hematocrit and serum albumin. These inflammatory biomarkers were positively associated with elevated serum phosphorous levels, an independent risk factor for CVD mortality in chronic kidney disease.

Evidence to support a role for long-chain n-3 fatty acids as a nutritional treatment for MIS is growing. Fish oil and long chain n-3 supplements are readily available over the counter and may provide an inexpensive way to modulate inflammation and clinical outcomes in MIS. Further research using larger sample sizes, earlier interventions and longer duration periods would seem to be warranted in patients with CKD.

\section{References}

1. Huang X, Lindholm B, Stenvinkel P, Carrero JJ (2013) Dietary fat modification in patients with chronic kidney disease: $\mathrm{n}-3$ fatty acids and beyond. J Nephrol 26: $960-974$

2. Gharekhani A, Khatami MR, Dashti-Khavidaki S, Razeghi E, Abdollahi A, et al (2014) Effects of oral supplementation with omega-3 fatty acids on nutritional state and inflammatory markers in maintenance hemodialysis patients. J Ren Nutr 24: 177-185.

3. Gharekhani A, Khatami MR, Dashti-Khavidaki S, Razeghi E, Noorbala AA et al. (2014) The effect of omega-3 fatty acids on depressive symptoms and inflammatory markers in maintenance hemodialysis patients: a randomized, placebo-controlled clinical trial. Eur J Clin Pharmacol 70: 655-665.

4. Gharekhani A, Khatami MR, Dashti-Khavidaki S, Razeghi E, Abdollahi A, et al. (2014) Potential effects of omega-3 fatty acids on anemia and inflammatory markers in maintenance hemodialysis patients. Daru 22: 11.

5. Hung AM, Booker C, Ellis CD, Siew ED, Graves AJ, et al. (2015) Omega-3 fatty acids inhibit the up-regulation of endothelial chemokines in maintenance hemodialysis patients. Nephrol Dial Transplant 30: 266-274.

6. Daud ZAM, Tubie B, QuaintonT, Osia R, Tubie S, et al. (2012) Effects of protein and omega-3 supplementation, provided during regular dialysis sessions, on nutritional and inflammatory indices in hemodialysis patients. Vascular Health and Risk Management 8: 187-195.

7. Ewers B, Riserus U, Marckmann P (2009) Effects of unsaturated fat dietary supplements on blood lipids, and on markers of malnutrition and inflammation in hemodialysis patients. J Ren Nutr 19: 401-411.

8. Poulia KA, Panagiotakos DB, Tourlede E, Rezou A, Stamatiadis D, et al. (2011) Omega-3 fatty acids supplementation does not affect serum lipids in chronic hemodialysis patients. J Ren Nutr 21: 479-484.

9. Harris MA, Lunn K, Quimby J, Kim S, Mulligan C (2012) Omega-3 fatty acids mitigate inflammation in felines with chronic kidney disease. FASEB J26: 43.1.

*Corresponding author: Mary Harris, Department of Food Science and Human Nutrition, Colorado State University, USA, Tel: 9704917462; E-mail: Mary.Harris@ColoState.edu

Received September 22, 2015; Accepted September 23, 2015; Published September 29, 2015

Citation: Harris M (2015) Is there a Role for Long Chain Omega-3 (N-3) Fatty Acids in Reversal of Malnutrition Inflammation Syndrome among Hemodialysis Patients?. Vitam Miner 3: e143.

Copyright: (c) 2015 Harris M. This is an open-access article distributed under the terms of the Creative Commons Attribution License, which permits unrestricted use, distribution, and reproduction in any medium, provided the original author and source are credited. 